\title{
BMJ Open Causal beliefs of mental illness and its impact on help-seeking attitudes: a cross-sectional study among university students in Singapore
}

\author{
Gregory Tee Hng Tan (D) ," Shazana Shahwan (D) , ${ }^{1}$ Chong Ming Janrius Goh, ${ }^{1}$ \\ Wei Jie Ong, ${ }^{1}$ Ellaisha Samari, ${ }^{1}$ Edimansyah Abdin, ${ }^{1}$ Kian Woon Kwok, ${ }^{2}$ \\ Siow Ann Chong, ${ }^{1}$ Mythily Subramaniam ${ }^{1}$
}

To cite: Tan GTH, Shahwan S, Goh CMJ, et al. Causal beliefs of mental illness and its impact on help-seeking attitudes: a cross-sectional study among university students in Singapore. BMJ Open 2020;10:e035818. doi:10.1136/ bmjopen-2019-035818

- Prepublication history and additional material for this paper are available online. To view these files, please visit the journal online (http://dx.doi. org/10.1136/bmjopen-2019035818).

Received 18 November 2019 Revised 29 May 2020 Accepted 05 June 2020

Check for updates

(C) Author(s) (or their employer(s)) 2020. Re-use permitted under CC BY-NC. No commercial re-use. See rights and permissions. Published by BMJ.

${ }^{1}$ Research, Institute of Mental Health, Singapore

${ }^{2} \mathrm{School}$ of Humanities and

Social Sciences, Division

of Sociology, Nanyang

Technological University,

Singapore

Correspondence to

Gregory Tee Hng Tan;

gregtth@gmail.com

\section{ABSTRACT}

Objectives A considerable proportion of those who suffer from mental illnesses in Singapore do not seek any form of professional help. The reluctance to seek professional help could be due to misconceptions about the causes of mental illnesses. Research has shown that help-seeking attitudes can predict actual service use. As young adults are most at risk of developing mental illnesses, this study aims to elucidate the impact of causal beliefs about mental illness on help-seeking attitudes among university students in Singapore.

Design Prior to attending an anti-stigma intervention, data on the Causal Beliefs about Mental IIIness, Inventory of Attitudes towards Seeking Mental Health services and questions pertaining to sociodemographic background were collected from participants using a self-administered questionnaire. Multiple linear regressions were performed to examine the relationship between causal beliefs and help-seeking, as well as their sociodemographic correlates. Settings A university in Singapore.

Participants 390 students who were studying in a University in Singapore.

Results Younger age was associated with higher scores on psychosocial attribution, while prior social contact with individuals with mental illness was significantly associated with lower scores on personality attribution. With regard to help-seeking attitudes; being a male and personality attribution were significantly associated with lower scores on 'Psychological Openness' and 'Indifference to Stigma', while psychosocial attribution was significantly associated with higher scores on 'Help-seeking Propensity'. Having prior social contact also predicted higher 'Psychological Openness', while being in Year 2 and 3 predicted lower scores on 'Indifference to Stigma'.

Conclusion Findings from this study suggest that helpseeking attitudes might be influenced by causal beliefs, with personality attribution being the most impairing. Hence, to reduce the wide treatment gap in Singapore, anti-stigma interventions targeting young people could focus on addressing beliefs that attribute mental illness to the personality of the individual.

\section{INTRODUCTION}

Mental disorders pose a greater global economic burden than chronic somatic
Strengths and limitations of this study

- A vignette-based approach was adopted to assess causal attributions relating to depression, which allows comparisons to other studies that also employed a vignette-based approach.

- This is the first study to examine the relationship between causal beliefs of mental illness and helpseeking attitudes using a sample of university students in Singapore.

- As only the depression vignette was used for this study, the findings for this study may not be generalisable towards other mental illnesses.

- As convenience sampling was adopted for this study, majority of participants recruited were Chinese and the study team was unable to recruit an adequate number of participants to represent the other major local ethnic groups in Singapore, to better reflect the influence of culture on causal beliefs and help-seeking.

- As the study is voluntary, there may be self-selection bias, as attitudes and knowledge of non-responders may differ from participants, which may not be possible to capture from this study.

diseases, with an estimated economic cost of US $\$ 2.5$ trillion worldwide, and this is expected to double by $2030 .{ }^{1}$ Despite the high prevalence of mental disorders and the considerable disease burden caused by it, many individuals with mental disorders remain untreated, resulting in a treatment gap that is wider than that in any other health sector. ${ }^{1}$ Furthermore, untreated mental disorders could result in severe ramifications for the individual. For instance, a person suffering from a single disorder-if not treated in time-may go on to develop more serious symptoms and other complex comorbid conditions that complicate the treatment regimen. ${ }^{23}$ 
Attitudes towards help-seeking, which includes the beliefs and willingness to seek help, have been shown to predict help-seeking behaviour. ${ }^{4}$ Given the implications of the wide treatment gap for mental disorders, it is important to understand the factors that influence an individual's help-seeking attitudes. Often, the underutilisation of mental health services can be due to stigma. ${ }^{56}$ Stigma is commonly defined as a denigrating attribute that brings about a mark of shame on the carrier, ${ }^{7}$ which typically leads to stereotype, prejudice and discrimination towards the individual. ${ }^{8}$ Stigma may impair an individual's attitudes towards seeking help insomuch that an individual may avoid seeking treatment due to their aversion towards being labelled with a mental illness (label avoidance) and its negative consequences, such as becoming ostracised or discriminated at work. ${ }^{9}$ Other factors that may influence help-seeking attitudes include the perceived need for seeking treatment from professionals as well as the perceived helpfulness of the treatment. ${ }^{1011}$

The causal belief of mental illness is another potential factor leading to stigma and the reluctance to seek help. ${ }^{12}{ }^{13}$ Importantly, an individual's treatment preferences may be influenced by the causal beliefs about their symptoms, which may in turn lead them to seek help from inappropriate sources or even refraining from seeking help altogether. ${ }^{14}{ }^{15}$ For instance, individuals attributing symptoms of mental illness to supernatural causes are less likely to view professional mental health services as effective, and are more likely to seek culturally traditional or religious forms of healing. ${ }^{16}$ A study by Bhikha et al (2005) suggests that lay beliefs about the causes of mental illness informs help-seeking choices; where the majority of participants who endorsed a dual explanatory model (both supernatural and biological causes) for psychosis took both the prescribed medication and followed the treatment prescribed by a faith healer. ${ }^{17}$

Rickwood et al (2005) proposed a model for the process of help-seeking for mental health problems among young people. According to this model, help-seeking begins with an individual's awareness or appraisal of problems before expression of one's symptoms and needs to others, and finally the willingness to seek help from available sources. ${ }^{18}$ Based on this model and the literature on the relationship between causal beliefs and help-seeking behaviours, ${ }^{11}{ }^{14-16}$ a hypothesis can be formulated, namely; aetiological beliefs of mental illness are probable determinants of help-seeking attitudes and intentions. Moreover, different causal attributions are likely to influence help-seeking behaviours and coping strategies towards mental illnesses differentially. Reducing the treatment gap, therefore, entails understanding and addressing the causal beliefs of mental illness in the context appropriate to the targeted audience.

The Singapore Mental Health Study conducted in 2016 found that the lifetime prevalence for mental disorders was $13.9 \%$. Depression was found to be the most prevalent condition in this study and youths emerged as the most vulnerable group for both lifetime and 12-month prevalence. ${ }^{19}$ The phenomenon of treatment gap is particularly relevant to Singapore, as the 12-month treatment gap for several mental illnesses in Singapore is high (78.6\%). Importantly, it has also been reported that those with higher education in Singapore are less likely to seek help. ${ }^{20}$ Since attitudes towards help-seeking predict actual service use, ${ }^{21}$ such findings highlight a need to understand the factors that affect help-seeking among young people with higher education in Singapore.

Previous studies in Singapore have investigated causal beliefs of mental illness where it was reported that psychosocial causes were the most likely to be attributed to mental disorders, followed by personality and physical causes. ${ }^{22}{ }^{23}$ However, these studies did not specifically sample young adults or investigate the relationship between causal beliefs and help-seeking attitudes. Research has shown that both causal beliefs and help-seeking attitudes can vary according to cultural and sociodemographic differences. ${ }^{14}{ }^{22-25}$ As depression is the most prevalent condition among the 18 to 34 age group in Singapore, understanding the causal beliefs of university students could be vital in reducing the treatment gap in this population.

In view of such findings, this study aims to examine the relationship between the causal beliefs about depression and help-seeking attitudes among university students in Singapore, taking into account their sociodemographic correlates.

\section{METHODS}

\section{Participants and procedures}

This study employed a convenience sampling method. Data for this study comprises the entire baseline data $(n=390)$ of the Advancing Research to Eliminate Mental Illness Stigma (ARTEMIS) study, which evaluated the effectiveness of an anti-stigma intervention. The ARTEMIS is an interventional study with repeated measures, and a total of 390 participants were recruited at baseline. Participants were first made to fill up a set of self-administered questionnaires prior to attending the intervention, to establish scores on baseline. After the intervention, the same 390 participants were then made to fill up another set of similar questionnaires, to allow evaluation of the intervention's efficacy. There was no attrition between baseline and post-intervention. To assess the lasting impacts of the intervention, participants were asked to complete a similar set of questionnaires 3 months from the date of intervention.

As the aim of this paper is purely to examine the relationship between causal beliefs of mental illness and helpseeking attitudes among Singapore's university students, only sociodemographic information and baseline data of all 390 participants from ARTEMIS collected prior to the intervention were used for the analysis in this study.

Participants for this study were recruited via an email invitation that was sent to students of various faculties within the university. Inclusion criteria were: (1) 
age between 18 to 35 years; (2) provision of informed consent, including parental consent for those below 21 as the age of majority in Singapore is 21 years; and (3) literacy in the English language. After providing written informed consent, participants were asked to fill up a series of self-administered questionnaires. The questionnaires were self-administered to minimise the effect of social desirability bias. The self-administered questionnaires included the Causal Beliefs about Mental Illness and the Inventory of Attitudes towards Seeking Mental Health Services (IASMHS). Sociodemographic information of the respondents was also captured using a short questionnaire.

\section{Instruments}

\section{Causal beliefs about mental illness}

The scale used in this study to assess causal beliefs about mental illness was originally developed by Reavley and Jorm. ${ }^{12} 23$ It comprises of 10 items describing the plausible causes of the problem depicted using a vignette approach. Participants had to first read the vignette which described an adult man with symptoms of depression (see online supplementary appendix A for more information on vignette) before responding to the items in the scale. Due to its cultural relevance, Pang et al (2018) adaptation of the causal beliefs scale was used in this study. A threefactor model was proposed for Pang et al (2018) adapted scale, and the factors were 'Physical', 'Personality' and 'Psychosocial'. Items that loaded on the 'Physical' factor comprised 'virus or infection' and 'allergy or reaction', while the 'Personality' factor consisted of 'being a nervous person' and 'having a weak character', and the 'Psychosocial'factor encompassed 'everyday problems such as stress, family arguments, difficulties at work or financial difficulties', 'recent death of a close friend or family', 'recent traumatic event such as a severe traffic accident' and 'childhood problems such as being badly treated or abused, losing one or both parents when young or coming from a broken home'.

\section{Inventory of Attitudes Towards Seeking Mental Health Services}

The IASMHS is a 24-items scale designed to measure an individual's attitudes towards seeking professional help for psychological issues and has been validated for use in a student population (IASMHS) ${ }^{26}$ Participants were asked to rate how much they agree or disagree with each of the items on a 5-point Likert scale ranging from 'disagree' to 'agree'. The original developer of the IASMHS identified a three-factor structure for the scale, namely 'Psychological Openness' (the degree of an individual's openness to acknowledging one's own psychological problems and to seek professional help for it), 'Help-seeking propensity' (the extent of one's willingness as well as perceived ability to seek professional psychological help) and 'Indifference to stigma' (how concerned an individual will be about others discovering them seeking professional help for their problems). Higher scores on a factor signify a more positive opinion towards that particular dimension of help-seeking attitude. This three-factor model of the IASMHS has also been validated recently by two other studies which employed different samples, ${ }^{27} 28$ suggesting that this factor-structure is rather robust.

\section{Analysis}

All analyses for this study were performed using SPSS V.23. Participants' responses to the items on the causal beliefs about mental illness scale were ranked and coded such that 'very likely' $=5$, 'likely' $=4$, ' depends' $=3$, 'unlikely'=2 and 'very unlikely'=1, while 'I don't know' responses were treated as missing data. Scores for each factor of the causal beliefs scale were derived by summing up the scores of items in each factor and dividing it by the number of items loaded on each respective factor. Higher scores on a factor represent stronger attribution to the factor as the cause of mental illness. Responses to the items in the IASMHS scale were also ranked and coded such that 'disagree' $=0$, 'somewhat disagree' $=1$, 'undecided' $=2$, 'somewhat agree' $=3$ and 'agree' $=4$. Scores for each factor in the IASMHS scale were generated by summing up the scores of items in each factor.

To understand the effects of sociodemographic characteristics on the causal attribution of mental illness, three multivariate linear regression models were run separately using each of the causal belief factors as dependent variables, while sociodemographic variables such as age, gender, ethnicity and year of study, were treated as independent variables. Other independent variables comprised social contact with a person(s) with mental illness such as having a close friend or family with mental illness and past experience in the mental health field. In order to elucidate the impact of causal attributions on help-seeking attitudes, three multivariate linear regression models were performed using each of the IASMHS factors as the dependent variable, while the aforementioned variables with the addition of causal beliefs factors were treated as independent variables. Data were analysed with the Statistical Package for Social Sciences V.23.0 (SPSS) and all statistically significant results are reported at $\mathrm{p}<0.05$.

\section{Patient and public involvement}

This research was done without patient involvement. No patients were involved in the planning or design of this study, nor consulted to develop patient relevant outcomes or interpret the results. No patients were invited to contribute to the writing or editing of this document for readability or accuracy.

\section{RESULTS}

\section{Descriptive analysis}

The demographic characteristics of our participants are shown in table 1 . The majority of participants were female $(60.3 \%)$ and of Chinese ethnicity $(82.8 \%)$. The mean $( \pm \mathrm{SD})$ age of the participants was $22.2 \pm 2.2$ years. In terms of years of study in the university, $33.3 \%$ were in 


\begin{tabular}{|c|c|c|}
\hline & Mean & SD \\
\hline \multirow[t]{2}{*}{ Age } & 22.2 & 2.2 \\
\hline & $\mathbf{N}$ & $\%$ \\
\hline \multicolumn{3}{|l|}{ Gender } \\
\hline Male & 155 & 39.7 \\
\hline Female & 235 & 60.3 \\
\hline \multicolumn{3}{|l|}{ Ethnicity } \\
\hline Chinese & 323 & 82.8 \\
\hline Non-Chinese & 67 & 17.2 \\
\hline \multicolumn{3}{|l|}{ Year of study } \\
\hline Year 1 & 130 & 33.3 \\
\hline Year 2 & 97 & 24.9 \\
\hline Year 3 & 79 & 20.3 \\
\hline Year 4 & 84 & 21.5 \\
\hline
\end{tabular}

Close friend or family member who has a mental illness

\begin{tabular}{lrr} 
Yes & 166 & 42.6 \\
No & 224 & 57.4 \\
$\begin{array}{l}\text { Past experience within mental health } \\
\text { field }\end{array}$ & \\
Yes & 86 & \\
No & 301 & 77.8 \\
\hline
\end{tabular}

Year 1, 24.9\% in Year 2, 20.3\% in Year 3 and $21.5 \%$ were in Year 4; and $42.6 \%$ of participants knew of at least one close friend or family member who had a mental illness, and $22.2 \%$ had past experience within the mental health field, while $0.5 \%(\mathrm{n}=2)$ had missing data for the variable past experience within the mental health field.

\section{Descriptive statistics on causal beliefs of depression}

Table 2 lists the mean score of each causal attribution of depression. The mean score for the three factors of causal beliefs were $1.8 \pm 0.8$ for physical attribution $(3.1 \%$ had missing data, $\mathrm{n}=12$ ), $4.2 \pm 0.5$ for psychosocial attribution (2.3\% had missing data, $\mathrm{n}=9$ ) and $3.1 \pm 1.0$ for personality attribution $(7.7 \%$ had missing data, $\mathrm{n}=30)$.

\section{Multivariate linear regressions of causal beliefs factors and help-seeking attitudes}

Results from multivariate linear regressions found no significant sociodemographic predictors for physical attribution of mental illness (table 3). On the other hand, younger age significantly predicted higher psychosocial

Table 2 Descriptive statistics on the causal beliefs of depression

\begin{tabular}{llll}
\hline & Physical & Psychosocial & Personality \\
\hline Mean (SD) & $1.8(0.8)$ & $4.2(0.5)$ & $3.1(1.0)$ \\
Range & $1.0-4.5$ & 2.0 to 5.0 & $1.0-5.0$ \\
\hline
\end{tabular}

attribution, while having close family or friends with mental illness or past experience in the mental health scene were both negatively associated with personality attribution.

With regards to help-seeking attitudes, multivariate linear regressions identified stronger psychosocial attribution to be associated with higher 'Help-seeking Propensity' whereas stronger personality attribution was linked to lower 'Psychological Openness' and 'Indifference to Stigma' (refer to table 4). In addition, being a male was significantly associated with poorer scores on 'Psychological Openness' and 'Indifference to Stigma', while participant's year of study and 'Indifference to Stigma' scores had an inverse relationship. Lastly, past experience in mental health field was significantly associated with both 'Psychological Openness' and 'Help-seeking Propensity' while having a close friend/relative with mental illness was only significantly associated with 'Psychological Openness' (refer to table 4). Missing data for 'Psychological Openness' factor was $0.5 \%(\mathrm{n}=2), 0.3 \%(\mathrm{n}=1)$ for 'Helpseeking Propensity' and $1.0 \%(\mathrm{n}=4)$ for 'Indifference to Stigma'.

\section{DISCUSSION}

Consistent with the findings of the study by Pang et al (2018), physical causes $(\mu=1.86 \pm 0.85)$ was the least endorsed attribution of depression among our participants, followed by personality causes $(\mu=3.18 \pm 1.00)$ and psychosocial causes $(\mu=4.25 \pm 0.57)$. Pang et al (2018) also found age differences in psychosocial beliefs, with older adults ( 35 to 49 years) being less likely than younger adults (18 to 34 years) to endorse psychosocial beliefs. Several studies have reported that endorsement of psychosocial causes of depression such as negative life events, broken homes and lack of parental affection, reduces stigma and the desire for social distance. ${ }^{29-31}$ Since causal attributions are linked to stigma ${ }^{912}$ and younger adults tend to show less stigma towards mental illness, ${ }^{3032}$ this possibly explains the age differences in psychosocial beliefs observed in our study. Taking into consideration the narrow age range of our participants, the age differences found in our study perhaps reflect the improvements of awareness initiatives that were integrated within the educational setting over the years.

With regards to help-seeking attitudes, our multiple linear regression analysis found several significant associations between subscales of IASMHS and sociodemographic factors, as well as between subscales of IASMHS and causal attribution of mental illness.

Having a close friend/family member with mental illness and past experience in the mental health field were both associated with lower scores on personality attribution, and this could be due to the effects of social contact with individuals who have a mental illness. Intergroup contact has been suggested to improve mental health literacy, increase empathy and reduce stigma. ${ }^{33}$ Hence, this finding may be tied to participants having a 


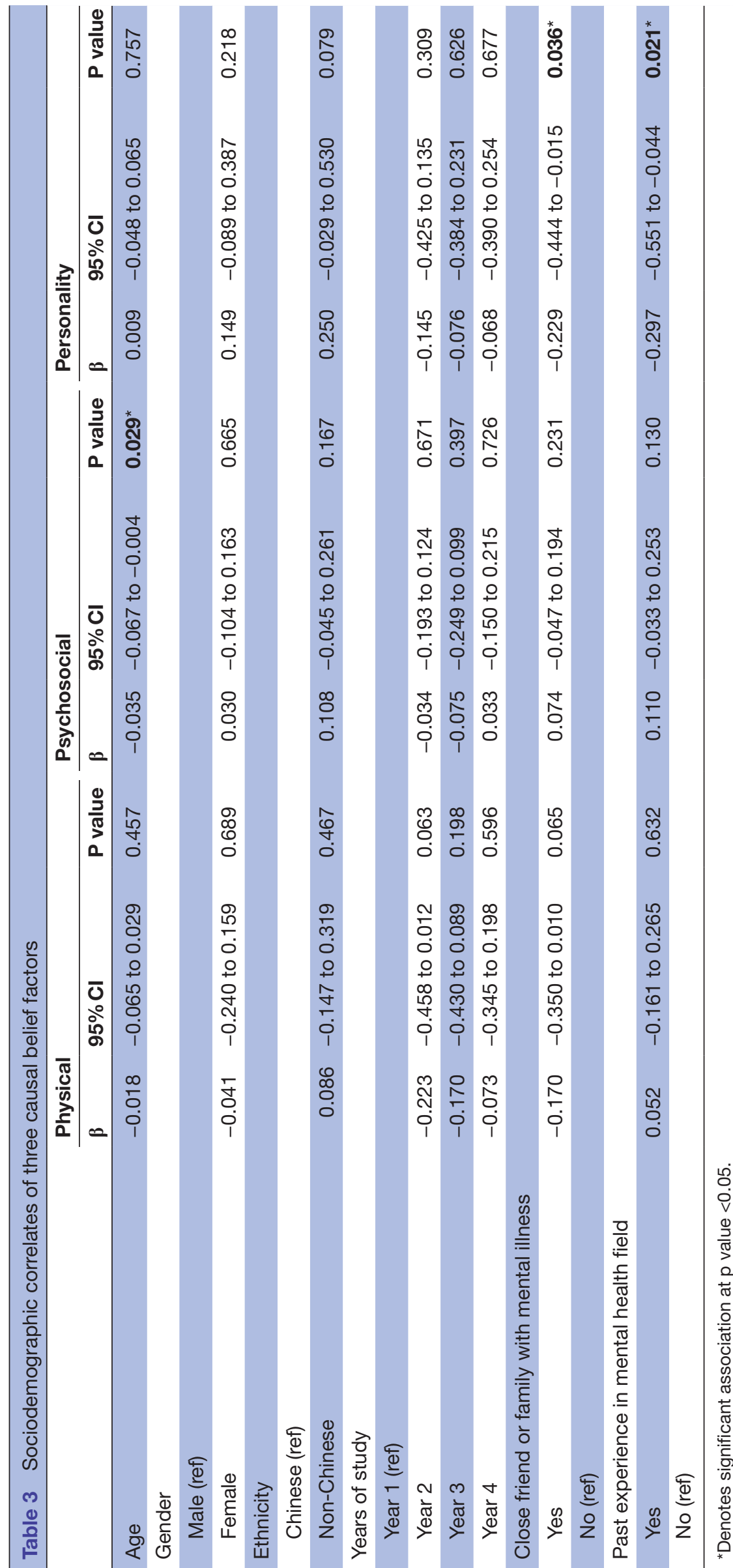

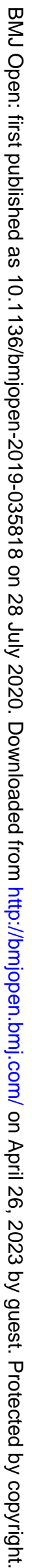




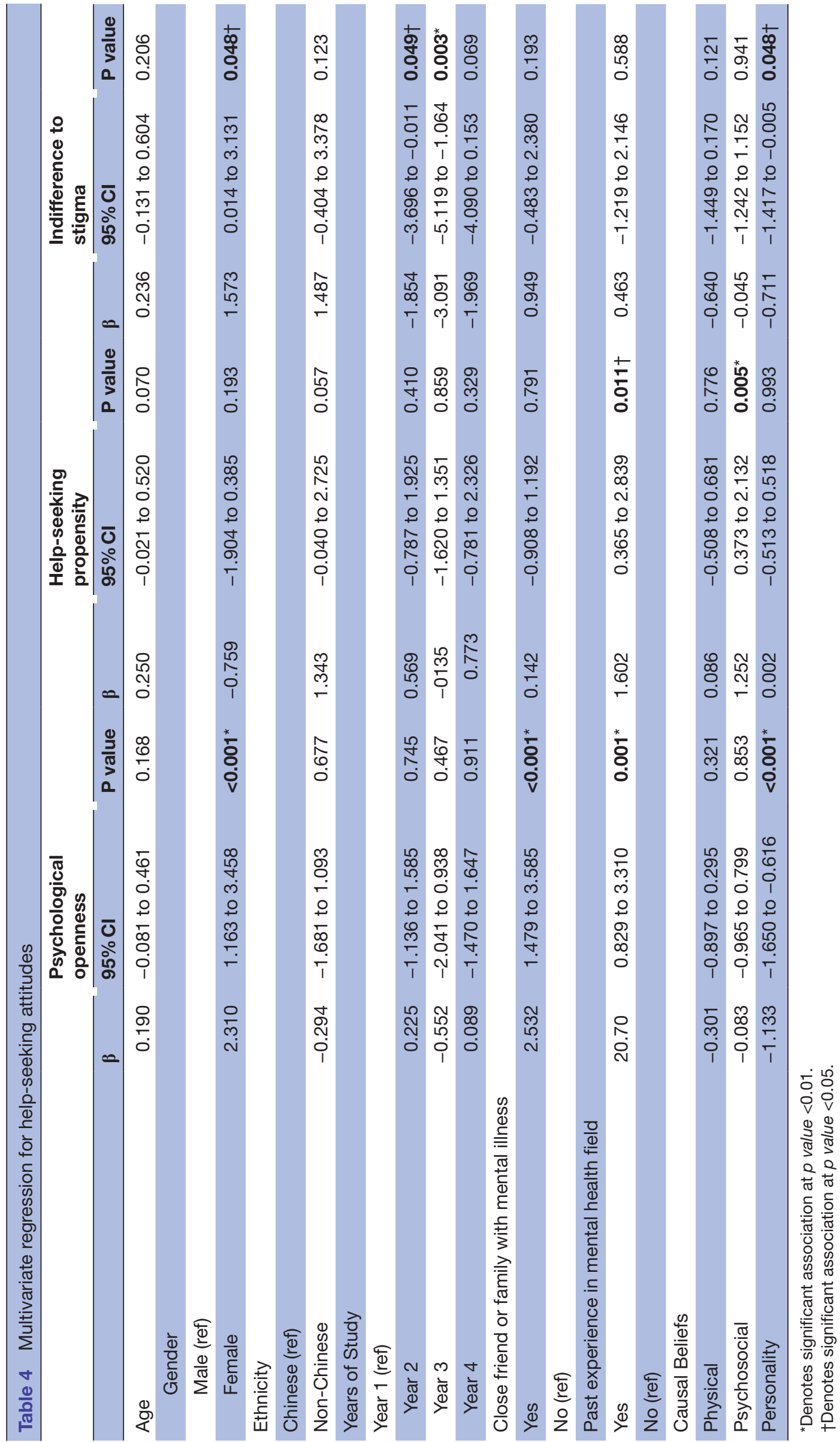

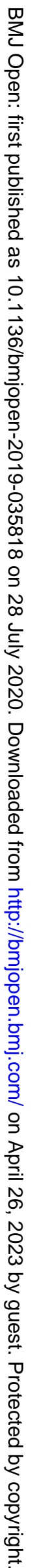


better understanding about the multifactorial causes of depression, and knowing that it is unlikely to be caused by just a 'weak or nervous personality'.

Interestingly, having a close friend/family with mental illness and past experience in the mental health field were also significantly correlated to 'Psychological Openness' scores, but only past experience in the mental health field was significantly correlated to 'Help-seeking Propensity'scores. A plausible explanation would be that intergroup contact helped to reduce stigma and thus promoted participant's willingness to acknowledge their psychological challenges and be open about it. However, 'Help-seeking Propensity' measures participant's knowledge of where to seek help and their perceived capability/ willingness to do so. In which case, intergroup contact with just friends/family with mental illness may not necessarily enhance their 'Help-seeking Propensity". On the other hand, having past experience in the mental health field would arguably improve their mental health literacy, thereby positively influencing their knowledge of where and when to seek help, as well as the perceived helpfulness of doing so. Nonetheless, further research may be needed to explore this phenomenon of how different forms of social contact affect help-seeking differentially.

Consistent with the extant literature, our study found a gender bias in help-seeking attitudes, ${ }^{25} 3435$ with females scoring significantly higher in 'Psychological Openness' and 'Indifference to Stigma' than their male counterparts. The gender differences in our findings might be due to cultural ideologies of masculinity ${ }^{36}$ where admitting that one has a mental health problem is akin to revealing one's weaknesses, and this is compounded by the perception that people who seek psychological treatment are often stereotyped as 'weak'. 'This possibly explains why males in the study were less open to acknowledging their psychological problems if any, and at the same time more apprehensive about others finding out should they seek treatment.

Our analysis also showed that attributing the cause of depression to an individual's personality was associated with lower 'Psychological Openness' and 'Indifference to Stigma'. This negative association between personality attribution and the two aforementioned help-seeking factors could be related to stigma. A study by Reavley and Jorm (2014) established that the belief in personality being a cause of mental illnesses is associated with higher personal weak not sick stigma. ${ }^{12}$ As such, attributing the cause of mental illness to a 'weak or nervous' personality is likely to engender denial towards one's condition, for it implies that mental illness is within a person's control (weak) and not a genuine medical issue (not sick). ${ }^{22}{ }^{37}$ This could result in self-blame and feelings of shame, which consequently leads to greater self-stigma and difficulty in acknowledging one's mental health problems, ${ }^{38-40}$ as well as greater consternation about others knowing should they seek treatment.

Personality attribution may also exacerbate the denial of one's condition especially in Asian cultures where having a mental illness is commonly seen as a disgrace to the family. A case in point would be the Chinese culture, where a mental illness is seen as a mark of shame that "tarnishes family honour, name and ancestors". ${ }^{41}$ In this case, personality attribution is likely to invoke feelings of blame and guilt on the sufferer for being unable to 'endure' one's problem, consequently fostering a strong antipathy towards acknowledging one's condition. Further, the belief that one can handle their symptoms on their own has been found to affect help-seeking intentions. ${ }^{101842}$ As such, the belief that mental illness is due to a 'weak or nervous' personality might inhibit an individual's openness to the idea of seeking help, especially so in cultures where seeking treatment is viewed as 'a lack of endurance, personality strength and dignity'. ${ }^{41}$ Conceivably, in such scenarios, individuals would face greater inertia in confronting their psychological issues (lower 'Psychological Openness') and also perceive greater stigma in seeking treatment (lower 'Indifference to Stigma').

In contrast, higher 'Help-seeking Propensity' scores were linked to greater endorsement of psychosocial attribution. The perceived need for psychological treatment from professionals and the perceived helpfulness of doing so has been shown to influence help-seeking attitudes ${ }^{10} 11$ with individuals who endorsed psychosocial attributions being more likely to regard it as a treatable condition, ${ }^{43}$ and are therefore more willing to seek treatment. An alternative explanation could be linked to how psychosocial causes (ie, childhood maltreatment and trauma) alludes to external factors that are beyond an individual's control. It has been shown that attribution of mental illness to external factors is positively associated with help-seeking, ${ }^{44}$ possibly because it evokes less self-afflicted blame and guilt within the individual. Furthermore, as mentioned above, several studies have reported that endorsement of psychosocial causes of depression reduces stigma and desire for social distance. ${ }^{29-31}$ Based on these findings, it can be postulated that when mental illness is attributed to psychosocial causes, individuals may be more willing to seek help as they perceive less stigma associated with the illness.

Another interesting finding is that as university students progress in their year of study in the school, their scores on 'Indifference to Stigma' tend to decrease. A possible explanation could be due to the fear of being labelled which in turn may lead to others regarding them as less competent. ${ }^{945}$ Thus, students may worry about their peers finding out that they are seeking treatment and thereby perceive them as weak or burdensome individuals who are unable to contribute sufficiently in projects/assignments. Alternatively, it may be that as students gradually progress in university, they tend to have larger social networks in school. Hence, there would be greater concern about others finding out that they are seeking treatment because of the stigma associated with the label ${ }^{45}$ and the fear of losing their social standing in school.

Similar findings in terms of the association between causal attribution of mental illnesses and help-seeking 
attitudes have been reported in previous studies. ${ }^{146}$ Chen and Mak's (2008) study, which also comprised undergraduate students, reported that help-seeking likelihood was positively correlated to attribution of mental illness to environmental/hereditary causes and negatively correlated to social-personal causes. ${ }^{14}$ This corroborates our study's finding that help-seeking attitudes are more likely to be unfavourable when the aetiological cause of mental illness is attributed to person-related reasons, whereas attributing mental illness to psychosocial reasons, likely encourages help-seeking. However, another study that investigated the relationship between causal beliefs of mental illness and help-seeking attitudes among a sample of persons with untreated depressive syndromes had slightly dissimilar results. ${ }^{46}$ The study found that lower attribution to person-related causes was linked to greater perceived need for help among participants with prior treatment experience. In contrast, attribution to biomedical causes was related to greater perceived need and stronger help-seeking intentions among participants without prior treatment experience. On the contrary, attributions to childhood trauma or stress-which were more related to our psychosocial factor-were not significantly associated with help-seeking in this study. A juxtapose of these two previous studies with the current one hence suggests that attribution of mental illness to person-related causes may have quite a universal impact on help-seeking, whereas the same may not be said for psychosocial causes.

Several studies which had also used the IASMHS demonstrated 'Help-seeking Propensity' to be the greatest factor out of the three to predict mental health service use. ${ }^{27} 2835$ Notably, it is recommended for future interventions targeting university students to emphasise more on psychosocial causes of mental illness, while addressing the beliefs that attribute mental illness to the personality of the individual, given its impairments on help-seeking attitudes. Importantly, our study also found that having social contact with individuals with mental illness reduces attribution of personality as a cause of mental illness. As such, it would be beneficial for future anti-stigma interventions or mental health literacy programmes to also incorporate social contact with people who have mental illness, in order to challenge misconceptions towards mental illnesses. ${ }^{33}$ In view of these considerations, and given that young people are most at risk of developing mental illness, ${ }^{19}$ there is perhaps a need for mental health literacy to be introduced earlier to youths.

There were some limitations to our study that are worth mentioning. Because our study used convenience sampling, we had to compare between two broad ethnic groups, that is, Chinese versus non-Chinese, as we did not have enough participants to represent the other major local ethnic groups (Malays and Indians). Hence, we were unable to elucidate the influence that other ethnic cultures may have on causal beliefs and help-seeking. The generalisability of our finding is another limitation. Since we used only the depression vignette in our study, our findings may not necessarily translate to other mental illnesses, and as our sample only comprised university students, our findings might not be indicative of youths in other settings. Lastly, the factor structure for the causal belief scale adopted in this study was generated from an exploratory factor analysis of a previous nationwide study in Singapore, which has yet to be validated in a university population which may have foreign students as well. Nonetheless, this factor structure was used as we believe it to be culturally relevant, given that the factor structure was generated locally, and Singapore happens to be a very small nation; moreover, the non-Singapore resident students constitute only a minority of the sample $(n=70)$.

Notwithstanding these limitations, findings from our study provide some useful insights into the relationship between causal beliefs and help-seeking attitudes among young people in university. Future similar research should target young people in other settings, and examine ethnic differences in causal beliefs and help-seeking behaviour. Also, future studies could look into other causal beliefs of mental illness (such as biogenetics and supernatural causes, which were not investigated in this study) and how stigma moderates the relationship between causal beliefs of mental illness and help-seeking attitude.

Findings from our study have several implications. First, the design of interventions to improve help-seeking attitudes among university students should consider the receptivity of male students, given that they have less positive help-seeking attitudes than their female counterparts. Second, our findings have shown the importance of causal beliefs on help-seeking attitudes. In order to mitigate the wide treatment gap among young people, future anti-stigma interventions should also focus on addressing the causes of mental illness. Finally, to better ameliorate the misconceptions regarding the causes of mental illness and correct stereotypes, anti-stigma interventions should also incorporate contact with an individual who has mental illness, and mental health literacy should be introduced to youths as early as possible.

Acknowledgements The authors would like to use this chance to thank the CHAT team for their help and educational material provided for the ARTEMIS intervention.

Contributors GTHT was responsible for writing the manuscript, conducting the fieldwork and statistical analysis. SS, CMJG, WJO and ES conducted the fieldwork and contributed to study's design. EA conducted power analysis and offered input to study's design. SAC, MS, and KWK contributed to study's design and supervised the overall study. All authors provided intellectual input to the manuscript and have given their final approval of the version to be published.

Funding The study was funded by Tote Board, Singapore.

Competing interests None Declared.

Patient consent for publication Not required.

Ethics approval Ethical approval was granted from the relevant institutional review board (National Healthcare Group, Domain Specific Review Board).

Provenance and peer review Not commissioned; externally peer reviewed.

Data availability statement Data are available upon reasonable request. Readers who wish to gain access to the data can write to the senior author MS @ mythily@ imh.com.sg to request access.

Open access This is an open access article distributed in accordance with the Creative Commons Attribution Non Commercial (CC BY-NC 4.0) license, which 
permits others to distribute, remix, adapt, build upon this work non-commercially, and license their derivative works on different terms, provided the original work is properly cited, appropriate credit is given, any changes made indicated, and the use is non-commercial. See: http://creativecommons.org/licenses/by-nc/4.0/.

\section{ORCID iDs}

Gregory Tee Hng Tan http://orcid.org/0000-0002-3262-741X

Shazana Shahwan http://orcid.org/0000-0003-4584-0751

\section{REFERENCES}

1 Trautmann S, Rehm J, Wittchen Hans-Ulrich, Wittchen $\mathrm{H}-\mathrm{U}$. The economic costs of mental disorders. EMBO Rep 2016;17:1245-9.

2 Kessler RC, Price RH. Primary prevention of secondary disorders: a proposal and agenda. Am J Community Psychol 1993;21:607-33.

3 McLaughlin CG. Delays in treatment for mental disorders and health insurance coverage. Health Serv Res 2004;39:221-4.

4 Gulliver A, Griffiths KM, Christensen H, et al. A systematic review of help-seeking interventions for depression, anxiety and general psychological distress. BMC Psychiatry 2012;12:81.

5 Gulliver A, Griffiths KM, Christensen H. Perceived barriers and facilitators to mental health help-seeking in young people: a systematic review. BMC Psychiatry 2010;10:113.

6 Jorm AF, Wright A, Morgan AJ. Where to seek help for a mental disorder? Med J Aust 2007;187:556-60.

7 Link BG, Phelan JC, Stigma C. Conceptualizing stigma. Annu Rev Sociol 2001;27:363-85.

8 Thornicroft G, Rose D, Kassam A, et al. Stigma: ignorance. prejudice or discrimination? The British journal of psychiatry : the journal of mental science 2007;190:192-3.

9 Corrigan P. How stigma interferes with mental health care. Am Psychol 2004;59:614-25.

10 Rughani J, Deane FP, Wilson CJ. Rural adolescents' help-seeking intentions for emotional problems: the influence of perceived benefits and stoicism. Aust J Rural Health 2011;19:64-9.

11 Edlund MJ, Unützer J, Curran GM. Perceived need for alcohol, drug, and mental health treatment. Soc Psychiatry Psychiatr Epidemiol 2006;41:480-7.

12 Reavley NJ, Jorm AF. Associations between beliefs about the causes of mental disorders and stigmatising attitudes: results of a national survey of the Australian public. Aust N Z J Psychiatry 2014:48:764-71.

13 Mannarini S, Rossi A. Assessing mental illness stigma: a complex issue. Front Psychol 2018;9:2722-22.

14 Chen SX, Mak WWS. Seeking professional help: etiology beliefs about mental illness across cultures. J Couns Psychol 2008;55:442-50.

15 Tompkins KA, Swift JK, Rousmaniere TG, et al. The relationship between clients' depression etiological beliefs and psychotherapy orientation preferences, expectations, and credibility beliefs. Psychotherapy 2017;54:201-206

16 Lauber C, Rössler W. Stigma towards people with mental illness in developing countries in Asia. Int Rev Psychiatry 2007;19:157-78.

17 Bhikha A, Farooq S, Chaudhry N, et al. Explanatory models of psychosis amongst British South Asians 2015.

18 Rickwood D, Deane F, Wilson C, et al. Young people's help-seeking for mental health problems 2005

19 Subramaniam M, Abdin E, Vaingankar J, et al. Tracking the mental health of a nation: prevalence and correlates of mental disorders in the second Singapore mental health study 2019.

20 Subramaniam M, Abdin E, Ajit Vaingankar J, et al. Minding the treatment gap: results of the Singapore mental health study 2019.

21 ten Have M, de Graaf R, Ormel J, et al. Are attitudes towards mental health help-seeking associated with service use? results from the European study of epidemiology of mental disorders. Soc Psychiatry Psychiatr Epidemiol 2010;45:153-63.

22 Picco L, Abdin E, Chong SA, et al. Attitudes toward seeking professional psychological help: factor structure and sociodemographic predictors. Front Psychol 2016;7:547-47.
23 Pang S, Subramaniam M, Lee SP, et al. The Singaporean public beliefs about the causes of mental illness: results from a multi-ethnic population-based study. Epidemiol Psychiatr Sci 2018;27:403-12.

24 Mojaverian T, Hashimoto T, Kim HS. Cultural differences in professional help seeking: a comparison of Japan and the U.S. Front Psychol 2012;3:615-15.

25 Nam SK, Chu HJ, Lee MK, et al. A meta-analysis of gender differences in attitudes toward seeking professional psychological help. J Am Coll Health 2010;59:110-6.

26 Mackenzie CS, Knox VJ, Gekoski WL, et al. An adaptation and extension of the attitudes toward seeking professional psychological help Scale1. J App/ Soc Psychol 2004;34:2410-33.

27 Hyland P, Boduszek D, Dhingra K, et al. A test of the inventory of attitudes towards seeking mental health services. Br J Guid Counc 2015;43:397-412.

28 Kantor V, Knefel M, Lueger-Schuster B. Investigating institutional abuse survivors' help-seeking attitudes with the inventory of attitudes towards seeking mental health services. Eur J Psychotraumatol 2017;8:1377528.

29 Dietrich S, Beck M, Bujantugs B, et al. The relationship between public causal beliefs and social distance toward mentally ill people. Aust N Z J Psychiatry 2004;38:348-54.

30 Jorm AF, Griffiths KM. The public's stigmatizing attitudes towards people with mental disorders: how important are biomedical conceptualizations? Acta Psychiatr Scand 2008;118:315-21.

31 Goldstein B, Rosselli F. Etiological paradigms of depression: the relationship between perceived causes, empowerment, treatment preferences, and stigma. J Ment Health 2003;12:551-63.

32 Subramaniam M, Abdin E, Picco L, et al. Stigma towards people with mental disorders and its components - a perspective from multiethnic Singapore. Epidemiol Psychiatr Sci 2017;26:371-82.

33 Gronholm PC, Henderson C, Deb T, et al. Interventions to reduce discrimination and stigma: the state of the art. Soc Psychiatry Psychiatr Epidemiol 2017;52:249-58.

34 Tedstone Doherty D, Kartalova-O'Doherty Y. Gender and selfreported mental health problems: predictors of help seeking from a general practitioner. Br J Health Psychol 2010;15:213-28.

35 Mackenzie CS, Gekoski WL, Knox VJ, Age KVJ. Age, gender, and the underutilization of mental health services: the influence of helpseeking attitudes. Aging Ment Health 2006;10:574-82.

36 Addis ME, Mahalik JR, Men MJR. Men, masculinity, and the contexts of help seeking. Am Psychol 2003;58:5-14.

37 Corrigan PW. Mental health stigma as social attribution: implications for research methods and attitude change. Clinical Psychology: Science and Practice 2000;7:48-67.

38 Corrigan PW, Druss BG, Perlick DA. The impact of mental illness stigma on seeking and participating in mental health care. Psychol Sci Public Interest 2014;15:37-70.

39 Corrigan PW, Rao D. On the self-stigma of mental illness: stages, disclosure, and strategies for change. Can J Psychiatry 2012;57:464-9.

40 Mak W, Wu C FM. Cognitive insight and causal attribution in the development of Self-Stigma among individuals with schizophrenia 2007

$41 \mathrm{Ng} \mathrm{CH}$. The stigma of mental illness in Asian cultures. Aust N Z J Psychiatry 1997;31:382-90.

42 Rickwood D, Deane F, Wilson C, et al. Young people's helpseeking for mental health problems. Australian e-Journal for the Advancement of Mental HealthAust 2005;4:1-34.

43 DCK L, Salkovskis PM, Warwick HMC. An experimental investigation of the impact of biological versus psychological explanations of the cause of "mental illness". J Ment Health 2005;14:453-64.

44 Kleinberg A, Aluoja A, Vasar V. Social support in depression: structural and functional factors, perceived control and help-seeking Epidemiol Psychiatr Sci 2013;22:345-53.

45 Overton SL, Medina SL. The stigma of mental illness. J Couns Dev 2008;86:143-51.

46 Stolzenburg S, Freitag S, Evans-Lacko S, et al. Individuals with currently untreated mental illness: causal beliefs and readiness to seek help. Epidemiol Psychiatr Sci 2019;28:446-57. 